\title{
Sex differences in metabolic rates in field crickets and their dipteran parasitoids
}

\author{
G. R. Kolluru · M. A. Chappell · M. Zuk
}

\begin{abstract}
Sex differences in metabolic rate (MR) can result from dimorphism in the performance of energetically demanding activities. Male crickets (Teleogryllus oceanicus) engage in costly calling and aggressive activity not performed by females. Consistent with this difference, we found higher maximal MR, factorial scope, and fat content in males than females. T. oceanicus song is also costly because it attracts the parasitoid fly Ormia ochracea. Parasitized crickets had reduced maximal MR consistent with a metabolic cost to harboring larvae. This cost was greater for females, either because females invest more heavily into reproduction at the expense of metabolic capacity, or because males are under stronger selection to respond to infection. Little is known about $O$. ochracea outside of its auditory system and parasitic lifestyle. We observed greater resting MR in male flies, possibly reflecting a sex difference in the requirement for metabolic power output, because male flies perform potentially costly mating behavior not seen in females. We found a positive relationship between larval density within a cricket and pupal resting MR, suggesting that crickets in good condition are able to both harbor more larvae and produce larvae with higher resting MR. These results reveal a complex interplay between the metabolism of crickets and their fly parasitoids.
\end{abstract}

Keywords Field cricket - Metabolic rate - Parasitoid fly · Pupal metabolism $\cdot$ Sexual dimorphism

\section{Introduction}

Sex differences in maximal metabolic power output are expected to evolve when one sex performs energetically demanding mate-searching or courtship activity more frequently or more intensely than the other sex (Chappell et al. 1996; Rogowitz and Chappell 2000; also see Krasnov et al. 2003, 2004). This expectation of sexual dimorphism in metabolic rate stems from the assumption that the sex engaging in the more costly behavior is selected to have a greater capacity to increase metabolic activity, if the capacity for activity is correlated with mating success (Rogowitz and Chappell 2000; Shillington and Peterson 2002). For example, male eucalyptusboring beetles (Phoracantha recurva and P. semipunctata) engage in vigorous mate-searching and fighting activities not performed by females, and, during forced terrestrial exercise, show higher maximal metabolic rates and aerobic scope than females (Rogowitz and Chappell 2000). Similarly, male rain beetles (Pleocoma species), which intensively search for mates both in flight and on the ground in very cold environments, demonstrate sustained endothermy not seen in females (Morgan 1987).

In this study, we examined sex differences in metabolic rates of Teleogryllus oceanicus, an Australian field cricket that has been introduced into Hawaii, and Ormia ochracea, an acoustically orienting tachinid fly parasitoid of T. oceanicus in Hawaii (Cade 1975; Zuk et al. 1993). Male field crickets engage in energetically expensive calling (Prestwich 1994) and fighting behavior (Burk 1983; Hack 1997a; Hofmann and Schildberger 2001) not performed by females. On this basis, we expected $T$. oceanicus males to be able to achieve higher maximal metabolic rates than females, at least for behaviors using the same muscle systems employed for calling and fighting (in Hawaiian populations, neither sex flies frequently). In contrast, the expectation for the parasitoid flies is not as clear. Although there is no airborne male acoustic display as in some other flies 
(e.g., Moulin et al. 2001; D. Robert and R. Hoy, personal communication), male ormiine flies exhibit hilltopping, defending and perching on conspicuous landmarks and attempting to clasp passing females (Lederhouse et al. 1976; Alcock and Smith 1995), and this flight behavior presumably has substantial energetic costs. On the other hand, female flies must perform energetically costly flights to search for patchily-distributed hosts (Müller and Robert 2001) while simultaneously producing and carrying large clutches of larvae (Roff 1977; Kolluru and Zuk 2001). Therefore, since both males and females must routinely engage in flight (the most energetically costly mode of animal locomotion), sex differences in fly metabolic rate are not expected to be predictably skewed toward one sex as in the crickets.

Despite widespread interest in host physiological responses to parasitoid infection (e.g., Dahlman and Herald 1971; Müller and Fritsche 1993; Alleyne et al. 1997), to our knowledge there have been no studies of the physiological interplay between crickets and $O$. ochracea with the exception of our recent study of male crickets (Kolluru et al. 2002). During their time in the host cricket, $O$. ochracea larvae consume muscle and fat body but not reproductive tissues (Adamo et al. 1995a). Parasitization reduces male and female cricket reproductive output (Adamo 1999; Kolluru et al. 2002) and shortens cricket lifespan (Simmons and Zuk 1994; Murray and Cade 1995). Crickets respond to the larvae by mounting an encapsulation response, attempting to surround the larvae with layers of hemocytes (Vinson 1990). Although the host encapsulation response is eventually used by larvae to construct a breathing tube (Vinson 1990), encapsulation of initial stage larvae may benefit the host by killing some larvae before they are large enough to usurp the encapsulation response to their own benefit.

We examined the possible effects of host traits on fly metabolism by measuring the aerobic metabolic rates of fly pupae and adults from hosts differing in the density of larvae. We also determined the influence of infection on cricket metabolism by measuring metabolic rates of unparasitized and naturally and experimentally parasitized crickets of both sexes. Using experimentally infected crickets allowed us to control for parasite load and ensured that we were measuring the metabolic rates of infected crickets (the infection status of a cricket cannot be determined pre-mortem). O. ochracea females release larvae onto the host and surrounding area, rather than injecting them into the host as do some hymenopterans (Belshaw 1994). Therefore, experimental infection closely mimics natural infection, with the exception that the hosts are anesthetized to prevent them from grooming the larvae off prior to larval ingress. We report the resting and maximal MR of crickets with and without parasites. Resting metabolic rate (RMR) indicates the energetic cost of inactive subsistence and is a lower limit to power output (Hack 1997b; Reinhold 1999), whereas maximal metabolic rate (MMR) indicates the upper limit to aerobic energy expenditure during a particular activity (Hinds et al. 1993). We addressed the following questions: (1) are there sex differences in RMR, MMR or factorial aerobic scope in crickets? Factorial aerobic scope (MMR/RMR) is frequently used as a measure of the ability of an animal to adjust its aerobic metabolism according to changing demands (Rogowitz and Chappell 2000). (2) Are there sex differences in the effects of parasitism on energy reserves (whole-body fat content) in crickets? (3) Because little is known about the reproductive biology of $O$. ochracea, we examined the developmental sequence of $O$. ochracea metabolic rates from hosts differing in larval density and asked whether there are sex differences in RMR in pupae or adult flies.

\section{Materials and methods}

\section{Experimental infection}

Parasitoid flies were trapped on the grounds of the University of Hawaii, Hilo using ceramic tiles coated with Tanglefoot insect trap coating and baited with tape-recorded, synthesized T. oceanicus song. Male and female crickets were anesthetized with $\mathrm{CO}_{2}(100 \%$ for approximately $10 \mathrm{~s}$ ) immediately before infection. Larvae were dissected from the flies and a dissecting pin was used to transfer three to five mobile larvae onto the membranous area around the front legs of each cricket (Cade 1984; Kolluru and Zuk 2001). This number of larvae is within the natural range found parasitizing the wild Hilo population of T. oceanicus (Zuk et al. 1995). As a control, another group of crickets was similarly anesthetized and handled but not infected. At least one parasitoid larva emerged from each parasitized cricket, and unparasitized crickets did not harbor any parasitoid larvae. As is the case in the wild, all infected crickets died within hours of larval emergence.

\section{Respirometry}

Our methods follow those of our previous study on cricket metabolic rates, and some RMR data for males presented here are taken from that study (Kolluru et al. 2002). In brief, we used both open- and closed-system respirometry to measure rates of $\mathrm{CO}_{2}$ production $\left(\dot{V} \mathrm{CO}_{2} ; \mathrm{ml} \mathrm{g}^{-1} \mathrm{~h}^{-1}\right)$ with a LiCor 6251 analyzer that resolved differences of $0.2-0.4 \mathrm{ppm}$ of $\mathrm{CO}_{2}$ in air. The validation for using $\dot{V} \mathrm{CO}_{2}$ as a metabolic index is given in Kolluru et al. (2002).

For closed-system measurements, we placed animals in containers (airtight 0.5 - or $0.3-1$ jars, or $60-$ or $140-\mathrm{ml}$ syringes, equipped with stopcock valves) which were flushed with dry, $\mathrm{CO}_{2}$-free air (scrubbed with Drierite and soda lime). Containers were then sealed for various durations to allow $\mathrm{CO}_{2}$ to accumulate. At the end of the measurement period, we injected samples of air from the containers through desiccant (magnesium perchlorate) 
into the LiCor 6251 . We computed $\dot{V} \mathrm{CO}_{2}$ from the final fractional $\mathrm{CO}_{2}$ concentration $\left(F \mathrm{ECO}_{2}\right)$, chamber volume $(V)$, and elapsed time $(\Delta t)$, with appropriate volume corrections to STP (standard temperature and pressure; $0^{\circ} \mathrm{C}$ and $101.3 \mathrm{kPa}$ ):

$$
\dot{V} \mathrm{CO}_{2}=\left(F \mathrm{ECO}_{2} \cdot V / \Delta t\right) /\left(1-F \mathrm{ECO}_{2}[1-(1 / \mathrm{RQ})]\right),
$$

where RQ is the respiratory quotient. We used an RQ of 0.85 ; due to the small value of $F \mathrm{ECO}_{2}(<0.003)$, variation in RQ between the minimum and maximum expected $(0.7-1.0)$ affects calculated $\dot{V} \mathrm{CO}_{2}$ by $<0.1 \%$.

Open-system measurements were made in 0.51 chambers with flow rates of dry, $\mathrm{CO}_{2}$-free air (80$200 \mathrm{ml} \mathrm{min}{ }^{-1}$ ), maintained at $\pm 1 \%$ by a Tylan mass flow controller. Chambers were kept at $30 \pm 0.5^{\circ} \mathrm{C}$ inside an environmental cabinet. Excurrent chamber air was dried (magnesium perchlorate) prior to entering the LiCor 6251. Instrument outputs were recorded every 2.5 or $4 \mathrm{~s}$ on Macintosh computers equipped with National Instruments $\mathrm{A} / \mathrm{D}$ converters and custom software ('Warthog Systems', www.warthog.ucr.edu). We calculated $\dot{V} \mathrm{CO}_{2}$ as:

$$
\dot{V} \mathrm{CO}_{2}=\dot{V}\left(F \mathrm{ECO}_{2}-F \mathrm{ICO}_{2}\right) /\left\{1-F \mathrm{ECO}_{2}[1-(1 / \mathrm{RQ})]\right\} \text {, }
$$

where $\dot{V}$ is flow rate corrected to STP and $\mathrm{FICO}_{2}$ is the initial fractional concentration of $\mathrm{CO}_{2}$ (zero in these experiments). As for closed-system measurements, we used an RQ of 0.85 , and as described for Eq. 1, variation in RQ has almost no effect on $\dot{V} \mathrm{CO}_{2}$ calculated from Eq. 2 due to the very low $\mathrm{FECO}_{2}(<0.002)$.

\section{Metabolic rates of crickets}

We used two approaches to determine cricket RMR. For lab-reared animals (descended from eggs collected in Hilo, Hawaii) we used open-system measurements, and RMR was determined as the mean minimal steadystate $\dot{V} \mathrm{CO}_{2}$ during periods of at least $10 \mathrm{~min}$ when activity (indicated by abrupt changes in $\dot{V} \mathrm{CO}_{2}$ ) was absent. Repeated RMR measurements were taken on these crickets once per day on days 2, 4, and 6 following infection of the parasitized crickets. Between measurements, crickets were housed in 0.5-1 plastic containers with shelter and ad libitum food (Fluker's cricket food, cat chow) and water, and were housed and tested at $30 \pm 1^{\circ} \mathrm{C}$.

As a control for different measurement techniques for RMR and maximal aerobic metabolism (see below), and to test for possible differences between lab-reared and field-captured animals, we used closed-system respirometry to determine RMR in wild-caught individuals from Hawaii. We collected crickets at night from lawns and other grassy areas, and measured RMR during the day (the inactive period for T. oceanicus). We monitored the metabolism chambers (0.3-1 jars or 60 - $\mathrm{ml}$ syringes) for activity, and data from animals observed to be active or heard calling were not used in RMR estimates. At the conclusion of measurements, crickets were euthanized by freezing and dissected to determine if they carried $O$. ochracea larvae.

We measured maximal aerobic metabolic rate (MMR) as $\dot{V} \mathrm{CO}_{2}$ during intense forced exercise for three different groups of crickets. Two of these groups were caught wild in Hilo, Hawaii. The first group consisted of individuals tested once either early (days 1-3) or late (days 4-5) in the course of infection. The second group was tested twice, on days 3 and 5 post-infection. The crickets were captured 3 days prior to testing and were housed in 0.5-1 plastic containers with shelter and ad libitum food (Fluker's cricket food, cat chow) and water. They were housed and tested at $27 \pm 1{ }^{\circ} \mathrm{C}$. Closed-system respirometry was used to determine MMR, with a 140$\mathrm{ml}$ syringe serving as the metabolism chamber. The syringe was sealed and shaken in a uniform motion for exactly $5 \mathrm{~min}$, forcing the cricket to exercise vigorously. At the conclusion of exercise, syringe air was injected through desiccant (magnesium perchlorate) into the $\mathrm{CO}_{2}$ analyzer. We computed $\dot{V} \mathrm{CO}_{2}$ according to Eq. 1 .

As a control for respirometric methods, we also determined MMR in a third group of 47 lab-reared, uninfected crickets using an open-flow system as described above for RMR. As for closed-system MMR estimates, crickets were exercised for $5 \mathrm{~min}$, and we used the highest 5-min continuous average of $\dot{V} \mathrm{CO}_{2}$ as MMR.

Analysis of cricket metabolic rate data

We constructed two separate ANOVA models to examine RMR variation in parasitized and unparasitized crickets. The first model, which was used to examine the RMR of lab-reared crickets tested repeatedly, was a repeated-measures ANOVA with sex and parasite status as fixed effects and day post infection as the repeated measure. The second model was used to examine data from the wild-caught crickets and the first trial for the lab-reared crickets, and incorporated sex, parasite status and source of crickets (lab-reared versus wild-caught) as main effects.

We examined variation in MMR using three ANOVA models. The first model incorporated sex, parasite status, and time period as fixed effects, and was used to examine data from wild-caught parasitized and unparasitized crickets tested singly. The second model incorporated sex and source of crickets (lab-reared versus wild-caught) as fixed effects and was employed to examine MMR of unparasitized lab-reared and wildcaught crickets together. The third model was a repeated-measures ANOVA designed to examine variation in MMR in the group of parasitized and unparasitized lab-reared crickets tested doubly, and incorporated sex and parasite status as fixed-effect terms, and day post infection as the repeated measure. 
Metabolic rate of flies

Resting metabolic rate (RMR) was measured for fly pupae that emerged from lab-reared Hawaii crickets. Another 11 larvae emerged from the crickets and pupated but did not eclose, and these were excluded from the results. Crickets were housed as described above. Pupae were housed in $0.5-1$ plastic containers at $30 \pm 1^{\circ} \mathrm{C}$ and subsequently tested at the same temperature, in a separate, modified 0.5-1 plastic container. Flies were housed and tested under the same conditions but with access to water in their housing containers. Four RMR measurements, each separated by approximately $24 \mathrm{~h}$, were made on each pupa, and one RMR measurement was made on each fly. Flies were weighed to the nearest $0.1 \mathrm{mg}$ and their RMR was measured within $24 \mathrm{~h}$ posteclosion. Pupal and fly RMR were measured blind with respect to sex. We were unable to obtain MMR data for flies.

Fat content of crickets

Whole-body fat content was determined for the male and female crickets from the wild-caught singly and doubly tested MMR groups described above (see Reznick and Braun 1987 for fat extraction protocol). Crickets whose wet masses were already known were killed and dried to a constant weight in a drying oven $\left(55^{\circ} \mathrm{C}\right)$. Parasitoid larvae were dissected out, and the crickets excluding the larvae were weighed to the nearest $0.01 \mathrm{mg}$ using a Cahn 21 electrobalance. Fat was extracted with petroleum ether for $96 \mathrm{~h}$ (in a series of four 24-h extractions) and the crickets were reweighed. Fat content was calculated by subtracting the lean dry mass from the fat dry mass, and is expressed as a percentage of the wet mass of the cricket on day 1 postinfection.

\section{Results}

Unless otherwise stated, all residuals were transformed to achieve normality and parametric tests were used to test for significance at $\propto=0.05$.

\section{Resting metabolic rate of crickets}

Female crickets were significantly heavier than males (ANOVA; $\quad F_{1,177}=9.00, \quad P=0.003$ ), and lab-reared crickets were heavier than field-caught crickets $\left(F_{1,177}=17.54, P<0.0001\right)$. There was no significant sex $\times$ cricket source interaction $\left(F_{1,177}=1.52, P=0.22\right)$. All crickets tested in the open-flow system yielded highquality RMR data, with periods of stable metabolism and inactivity lasting many minutes. Given the high resolution of the $\mathrm{CO}_{2}$ analyzer (better than $0.5 \mathrm{ppm}$ $\mathrm{CO}_{2}$ ) compared to the metabolism-induced change in $\mathrm{CO}_{2}$ concentration (usually $>100 \mathrm{ppm}$ ), we were easily
Table 1 Analysis of variation in cricket resting metabolic rate as a function of sex, infection status, day post-infection and source (wild-caught or lab-reared) of crickets. Values are $F_{(\mathrm{df})} ; P$

\begin{tabular}{lll}
\hline & $\begin{array}{l}\text { Model I: } \\
\text { lab-reared, } \\
\text { tested singly }\end{array}$ & $\begin{array}{l}\text { Model II: trial 1 } \\
\text { for lab-reared } \\
\text { versus field-caught, } \\
\text { tested singly }\end{array}$ \\
\hline Sex & $3.36_{(1,31)} ; 0.077$ & $1.16_{(1,174)} ; 0.69$ \\
Infection status & $1.93_{(1,31)} ; 0.17$ & $2.13_{(1,174)} ; 0.15$ \\
Sex $\times$ Infection status & $0.91_{(1,31)} ; 0.35$ & $0.29_{(1,174)} ; 0.59$ \\
Day & $1.43_{(2,31)} ; 0.26$ & - \\
Source of crickets & - & $0.78_{(1,174)} ; 0.38$ \\
Sex $\times$ Source of crickets & - & $0.15_{(1,174)} ; 0.70$ \\
Infection status $\times$ & - & $0.10_{(1,174)} ; 0.75$ \\
Source of crickets & & \\
\hline
\end{tabular}

able to resolve activity-induced fluctuations in metabolic rate. Similarly, most of the animals measured in closed systems remained quiet and provided good RMR data.

Male and female crickets did not differ significantly in numbers of parasitoid larvae per cricket (ANOVA; $\left.F_{1,38}=1.29, P=0.26\right)$, nor did lab-reared and wildcaught crickets $\left(F_{1,38}=0.03, P=0.87\right)$. Model I revealed no significant effects of sex, infection status, or day postinfection on RMR, and no significant sex $\times$ infection status interaction (Table 1). Model II likewise revealed no main effects of sex or infection status on RMR, and revealed no significant difference in RMR between labreared and wild-caught crickets (Table 1).

Maximal metabolic rate of crickets

\section{Singly exercised groups}

Female crickets were significantly heavier than males (ANOVA: $F_{1,94}=19.20, P<0.0001$ ), and lab-reared crickets were heavier than wild-caught crickets $\left(F_{1,94}=41.24, P<0.0001\right)$. There was no significant interaction between sex and source of crickets $\left(F_{1.94}=0.02\right.$, $P=0.90)$. Male and female cricket parasite loads did not differ significantly ( $t$-test: $t=0.72, P=0.48)$.

Model I showed that male crickets had significantly higher MMR than females, and that unparasitized crickets had significantly higher MMR than parasitized crickets (Table 2, Fig. 1). There was also a significant sex $\times$ infection status interaction because the difference in MMR between parasitized and unparasitized females was greater than the difference between parasitized and unparasitized males. There was no significant time period effect, nor was there a sex $\times$ time period interaction (Table 2). There was a significant infection status $\times$ time interaction, resulting from the greater difference in MMR between unparasitized and parasitized crickets late in the course of infection compared to early. The three way interaction was not significant (Table 2). Model II also revealed that male crickets had higher MMR than female crickets, and that field-caught 
Table 2 Analysis of variation in cricket maximal metabolic rate as a function of sex, infection status, time period or day postinfection and source (wildcaught or lab-reared) of crickets. Values are $F_{(\mathrm{df})} ; P$

\begin{tabular}{llll}
\hline & $\begin{array}{l}\text { Model I: } \\
\text { wild-caught, tested singly }\end{array}$ & $\begin{array}{l}\text { Model II: } \\
\text { Lab-reared versus } \\
\text { wild-caught, } \\
\text { tested singly }\end{array}$ & $\begin{array}{l}\text { Model III: } \\
\text { lab-reared, } \\
\text { tested doubly }\end{array}$ \\
\hline Sex & $54.94_{(1,63)} ;<0.0001^{*}$ & $5.42_{(1,70)} ; 0.023^{*}$ & $0.54_{(1,21)} ; 0.47$ \\
Status & $20.25_{(1,63)} ;<0.0001^{*}$ & - & $1.01_{(1,21)} ; 0.33$ \\
Sex $\times$ status & $6.11_{(1,63)} ; 0.017^{*}$ & - & 0.02 \\
Time period or day & $1.71_{(1,63)} ; 0.20$ & - & $0.27_{(1,21)} ; 0.61$ \\
Sex $\times$ time period & $2.79_{(1,63)} ; 0.10$ & - & - \\
Status $\times$ time period & $7.06_{(1,63)} ; 0.01^{*}$ & - & - \\
Sex $\times$ status $\times$ time period & $0.27_{(1,63)} ; 0.61$ & - & - \\
Source of crickets & - & $8.44_{(1,70)} ; 0.005^{*}$ & - \\
Sex $\times$ source of crickets & - & $0.19_{(1,70)} ; 0.66$ & - \\
\hline
\end{tabular}

crickets had somewhat higher MMR than lab-reared crickets (Fig. 1, Table 2).

Among singly exercised crickets in this MMR group, males had a higher fat content than females (ANOVA: $\left.F_{1,61}=4.35, P=0.041\right)$. Fat content did not vary with the day post-infection of the measurement, or infection status (Fig. 2); however, there was a marginally nonsignificant sex $\times$ infection status interaction, because the difference in fat content between parasitized and unparasitized females was slightly greater than the difference between parasitized and unparasitized males.

\section{Doubly exercised group}

In this wild-caught group of crickets, females and males did not differ in mass, although the result was marginally non-significant in favor of females ( $t$-test: $t=2.07$, $P=0.050)$. Male and female cricket parasite loads did not differ significantly ( $t$-test: $t=0.35, P=0.73)$. Model
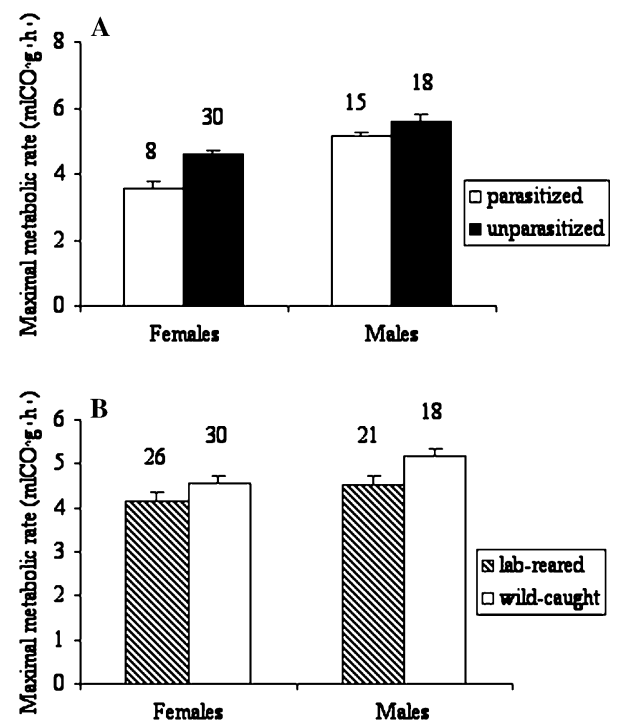

Fig. 1 Maximal metabolic rates of Teleogryllus oceanicus crickets tested singly as a function of sex and parasite status (A) and sex and source (B). Numbers above the bars indicate sample sizes. Values are corrected to $30^{\circ} \mathrm{C}$. Statistical analysis of these results is given in Table 2. Bars show mean $+\mathrm{SE}$
III revealed no significant effects of sex, infection status, the interaction between the two, or day post-infection on MMR (Table 2).

In this doubly exercised group of crickets, fat content was much lower than in the singly exercised group (Fig. 2). There was no sex difference, infection status effect, or infection status $\times$ sex interaction in fat content for this group.

\section{Factorial scope}

We calculated factorial scope as MMR/RMR (Rogowitz and Chappell 2000) for unparasitized and parasitized male and female crickets $(n=5$ parasitized females, 24 unparasitized females, 15 parasitized males, and 18 unparasitized males). The RMR and MMR data were collected from different but size-matched individuals. Males had slightly higher aerobic scope than females, though the difference was marginally non-significant (ANOVA; $F_{1,58}=3.82, P=0.056$ ). Unparasitized crick-

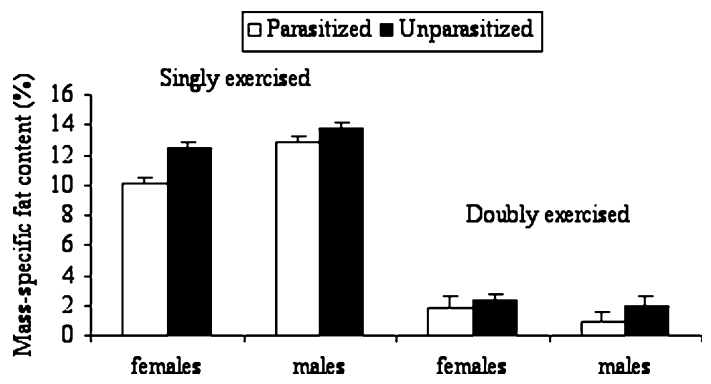

Fig. 2 Mass-specific whole-body fat content of wild-caught unparasitized and parasitized female and male crickets that were exercised either singly or doubly post-infection. Numbers above the bars indicate sample sizes. Parasitized crickets each harbored 3 5 Ormia ochracea larvae. Fat contents exclude parasitoid larvae. In the doubly exercised group, there was no significant effect of sex $\left(F_{1,20}=2.28, P=0.15\right)$, parasite status $\left(F_{1,20}=3.40, P=0.08\right)$, or status $\times$ sex $\left(F_{1,20}=0.26, P=0.61\right)$. In the singly exercised group, there was no significant day post-infection $\left(F_{1,61}=0.001, P=0.97\right)$ or infection status $\left(F_{1,61}=0.08, P=0.78\right)$ effect. There was a significant effect of $\operatorname{sex}\left(F_{1,61}=4.35, P=0.041\right)$. The difference between parasitized and unparasitized crickets was slightly greater for females than males [marginally non-significant sex $\times$ infection status term; $\left.\left(F_{1,61}=3.90, P=0.053\right)\right]$. None of the other interaction terms was significant (all $P>0.10$ ). Bars show mean $+\mathrm{SE}$ 


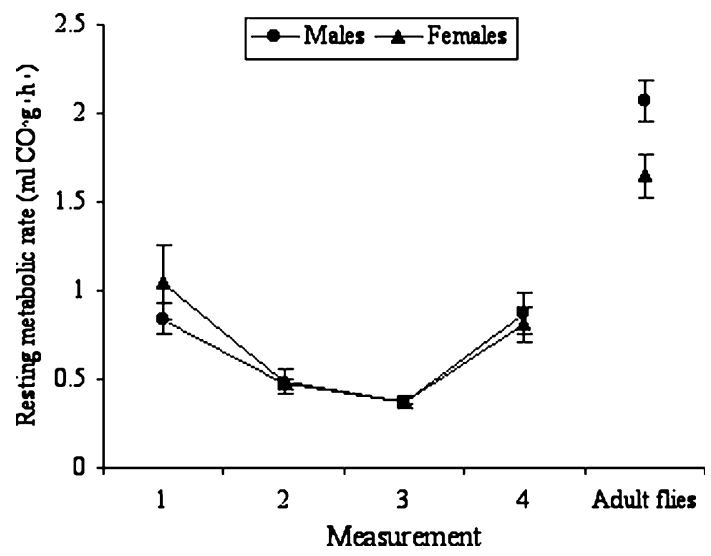

Fig. 3 Resting metabolic rates (RMR) of male $(n=14)$ and female $(n=9)$ Ormia ochracea pupae and newly eclosed adults. The sequential measurements on pupae were made approximately $24 \mathrm{~h}$ apart. Male and female pupae did not differ in RMR (repeatedmeasures analysis of covariance; $F_{1,19}=1.91, P=0.18$ ). Male flies had significantly higher RMR than female flies (ANCOVA: $\left.F_{1,20}=5.64, P=0.028\right)$. Values are corrected to $30^{\circ} \mathrm{C}$. Points show mean $\pm \mathrm{SE}$

ets had significantly higher scope than parasitized crickets $\left(F_{1,58}=4.77, P=0.033\right)$. There was a significant sex $\times$ infection status interaction $\left(F_{1,58}=4.18, P=0.046\right)$ because the difference between infected and uninfected crickets was greater for females than for males.

\section{Metabolic rate of flies}

Male flies had significantly higher RMR than female flies (Fig. 3). RMR of developing flies followed a Ushaped trajectory over time, with larvae, early pupae and newly eclosed adult flies having higher metabolic rates than late pupae (Fig. 3). Male and female pupae did not differ in RMR, but RMR varied positively with the density of larvae per host (Fig. 4). There was no influence of density of larvae within a host on adult fly RMR.

\section{Discussion}

We found significant sex differences in exercise energy metabolism in Teleogryllus oceanicus crickets consistent with the prediction that male crickets have a greater capacity for metabolic power output and ability to increase metabolic activity during forced locomotion than females. Males had higher maximal metabolic rates (MR) than females in both the wild-caught and labreared singly-tested groups, greater factorial scope (the ability to change aerobic MR; Hinds et al. 1993), and a higher whole-body fat content. Female crickets, selected to invest heavily in egg production (Honek 1993), are presumably not under selection for combat capacity or calling signal strength like males, and are less able to increase metabolic activity during intense terrestrial exercise (Chappell et al. 1996; Rogowitz and Chappell 2000). Consistent with this explanation, singly exercised, wild-caught female crickets had higher body mass than males, but did not differ from males in mass-specific resting MR.

Unparasitized crickets had significantly higher maximal MR (in the singly tested group) and factorial scope than parasitized crickets. In agreement with our prediction that male and female crickets would differ in the effects of parasitoid infection on metabolism, we found a significantly greater reduction in maximal MR and factorial scope with parasitism in females than in males, even though there was no sex difference in the number of larvae per host. This suggests that the adverse metabolic effects of harboring parasitoid larvae were greater for females than for males. One potential explanation for these results is based on the observation that male $T$. oceanicus in Hawaii are parasitized far more frequently than females $(<7 \%$ of females were parasitized in one field survey in Hilo, Hawaii, versus approximately $18 \%$ of males; Zuk et al. 1993). Males may therefore be subject to more intense selection by $O$. ochracea, such that they are better able to overcome the adverse effects of parasitism. For example, males may have evolved a greater ability to efficiently encapsulate parasitoid larvae without compromising metabolic capacity. Alternatively, female crickets may have a lower ability to respond to parasitism because they are selected to invest more heavily into reproduction (e.g., egg production) than into immunity. This is supported by recent findings that food-deprived $T$. oceanicus males had greater encapsulation ability than food-deprived females, a difference not seen in animals fed ad libitum (Zuk et al. 2004).
Fig. 4 RMR of O. ochracea pupae as a function of larval density (number of larvae per gram of cricket). Sample sizes are as described in the caption to Fig. 3. RMR varied positively with larval density $\left(F_{1,19}=14.54, P=0.001\right)$. There was no influence of larval density on eclosed fly RMR $\left(F_{1,20}=0.003, P=0.96\right)$
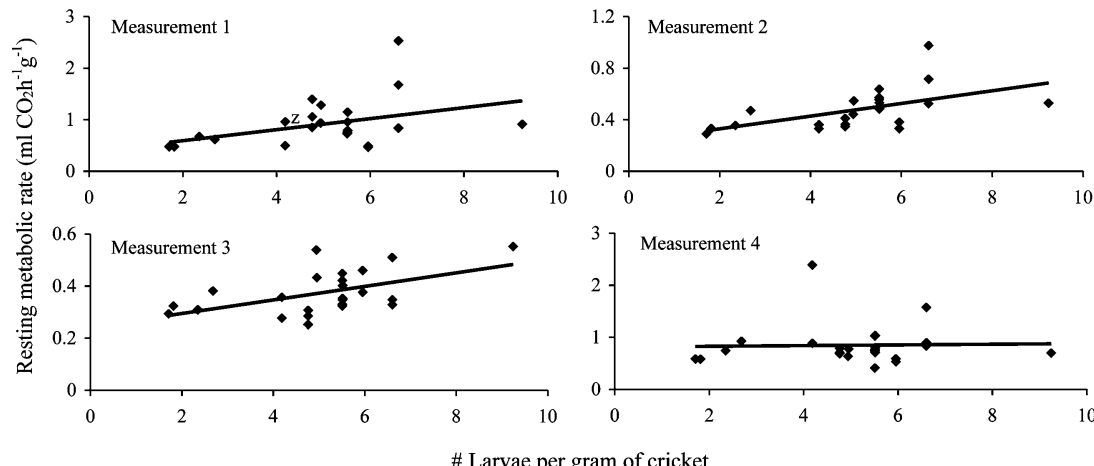
Two other aspects of our cricket metabolism findings merit additional comment. First, there was no difference in resting metabolism between field-caught versus lab-reared crickets, but maximal MR was significantly lower in lab-reared crickets than in wild-caught crickets. This may have resulted from differences in measurement protocol, or from differences in the crickets themselves (although the lab-reared animals were descended from eggs collected from the same Hawaiian populations from which we obtained field animals). The consistent resting MR in field-caught and lab-reared animals suggests (although not conclusively) no differences between the two stocks. We feel that measurement differences are the most likely explanation: forced exercise $(5 \mathrm{~min}$ of shaking the chamber) was employed to elicit maximal MR in both stocks, but the wild-caught animals were tested in closed containers and the lab-reared animals in an open-flow system. Different individuals performed the measurements on the two stocks and the wild-caught animals may have been exercised more vigorously.

The second puzzling observation is the much lower fat content of doubly exercised crickets than of singly exercised crickets. The fat mass difference is far larger than can be accounted for by the additional metabolic expenditures during the second 5-min exercise bout. It is more likely that behavioral or metabolic changes following the initial exercise test (i.e., higher resting MR, greater activity, or lower food intake) account for an apparently greater draw-down of lipid stores in doubly exercised animals.

As in our previous study (Kolluru et al. 2002), we found no apparent effect of parasitoid larvae on resting MR in either sex of crickets. However, it is probable that parasitoid larvae contributed a substantial fraction of the total resting MR of the host-parasitoid complex (Kolluru et al. 2002; see also Alleyne et al. 1997). If we assume that the MR of newly-emerged larvae (Fig. 3) is similar to that of pre-emergence larvae, then parasitized crickets close to the time of parasite emergence have substantially lower resting MR than our data indicate. This suggests that there is a sizeable reduction of substrate turnover (and hence ATP production) in crickets harboring $O$. ochracea larvae - unsurprising given the consumption of host tissue by these parasitoids (Adamo et al. 1995a) - and that this cost may be responsible for the decline in reproductive effort seen in infected male crickets (Kolluru et al. 2002). This is at least partly consistent with other studies of parasitoid effects on host metabolism (Dahlman and Herald 1971; Alleyne et al. 1997; Rivers and Denlinger 1994). However, such studies almost exclusively involve hymenopteran parasitoids, which differ markedly from tachinids because they envenomate (and in some cases paralyze) their hosts (Godfray 1994).

We also found significant sex differences in resting MR in the parasitoid flies. Eclosed male flies had higher resting MR than females, a difference not seen in the developing pupae. We speculate that this difference may reflect greater selection on males to rapidly produce
ATP, because they engage in energetically costly reproductive behavior not performed by females. Further studies into the reproductive biology of these flies, including the ecological and energetic expenses involved in hilltopping by males and in host-searching by females, are needed to determine whether one sex assumes more costs than the other. Our data on the resting MR of developing fly pupae are in agreement with previous studies of other insect pupae in showing a U-shaped temporal response, with newly-pupated individuals and late-stage pupae producing higher levels of $\mathrm{CO}_{2}$ than pupae of intermediate age (Sayle 1928; Odell 1998). The resting MR of adult flies was also within the range found in other studies of tachinid flies (Chappell and Morgan 1987; Alekseeva et al. 1993).

Parasitoid reproductive success is dependent on host traits such as body size and the number and size of competing parasites (Speirs et al. 1991; Rivers and Denlinger 1994; Hunt and Allen 2000). Despite the adverse effects of superparasitism (presence of multiple clutches within one host) on $O$. ochracea (Adamo et al. 1995b), these flies do not avoid previously infected hosts (Adamo et al. 1995b). Furthermore, there is no direct relationship between host size and fly clutch size (Adamo et al. 1995b; Kolluru and Zuk 2001). This suggests that female flies do not alter larviposition behavior based on cricket condition, even though smaller parasitoid clutches yield larger flies with higher fitness (Adamo et al. 1995b; Kolluru and Zuk 2001). Given the data on within-host competition among parasitoids, resting MR is a potentially important aspect of fly fitness that might be expected to vary with the number of larvae in the host during fly development. Surprisingly, we found a positive influence of larval density (number of larvae per gram of cricket) on the resting MR of adult flies. Although this result appears counterintuitive from the perspective of parasitoid competition for host resources, it is consistent with the idea that crickets in better condition are both able to sustain higher larval densities and able to yield pupae with greater resting MR.

Acknowledgements We are grateful to J.T. Rotenberry for assistance with field measurements, L.P. Nunney, D. Price and D.N Reznick for allowing us to use their laboratory facilities, and the University of Hawaii, Hilo groundskeeping staff for their assistance in the field. This research was supported by the University of California at Riverside Intramural Funds to M.A.C. M.Z. is supported by grants from the US National Science Foundation and the UCR Academic Senate. The experiments comply with the current laws of the USA.

\section{References}

Adamo SA, Robert D, Hoy RR (1995a) Effects of a tachinid parasitoid, Ormia ochracea, on the behaviour and reproduction of its male and female field cricket hosts (Gryllus spp). J Insect Physiol 41:269-277

Adamo SA, Robert D, Perez J, Hoy RR (1995b) The response of an insect parasitoid, Ormia ochracea (Tachinidae), to the 
uncertainty of larval success during infestation. Behav Ecol Sociobiol 36:111-118

Adamo SA (1999) Evidence for adaptive changes in egg laying in crickets exposed to bacteria and parasites. Anim Behav 57:117124

Alcock J, Smith AP (1995) Landmark-defense and scramble competition mating systems in two Australian tachinid flies (Diptera). J Kansas Entomol Soc 68:85-94

Alekseeva TA, Zotin AI, Yasyukevich VV, Sidorova NV (1993) Oxygen uptake by adult forms of the Diptera. Izv Ross Akad Nauk 3:426-435

Alleyne M, Chappell MA, Gelman DB, Beckage NE (1997) Effects of parasitism by the braconid wasp Cotesia congregata on metabolic rate in host larvae of the tobacco hornworm, Manduca sexta. J Insect Physiol 43:143-154

Belshaw R (1994) Life history characteristics of Tachinidae (Diptera) and their effect on polyphagy. In: Hawkins BA, Sheehan W (eds) Parasitoid community ecology. Oxford University Press, Oxford, pp 145-162

Burk T (1983) Male aggression and female choice in a field cricket (Teleogryllus oceanicus): the importance of courtship song. In: Gwynne DT, Morris GK (eds) Orthopteran mating systems: sexual competition in a diverse group of insects. Westview, Boulder, Colorado, pp 97-119

Cade WH (1975) Acoustically orienting parasitoids: fly phonotaxis to cricket song. Science 190:1312-1313

Cade WH (1984) Genetic variation underlying sexual behavior and reproduction. Am Zool 24:355-366

Chappell MA, Morgan KR (1987) Temperature regulation, endothermy, resting metabolism, and flight energetics of tachinid flies (Nowickia sp.). Physiol Zool 60:550-559

Chappell MA, Zuk M, Johnsen TS (1996) Repeatability of aerobic performance in red junglefowl: effects of ontogeny and nematode infection. Funct Ecol 10:578-585

Dahlman DL, Herald F (1971) Effects of the parasite, Apanteles congregatus, on respiration of tobacco hornworm, Manduca sexta larvae. Comp Biochem Physiol A 40:871-880

Godfray HCJ (1994) Parasitoids: behavioral ecology and evolutionary ecology. Princeton University Press, Princeton, New Jersey

Hack MA (1997a) The energetic costs of fighting in the house cricket, Acheta domesticus L. Behav Ecol 8:28-36

Hack MA (1997b) The effects of mass and age on standard metabolic rate in house crickets. Physiol Entomol 22:325-331

Hinds DS, Baudinette RV, MacMillen RE, Halpern EA (1993) Maximum metabolism and the aerobic factorial scope of endotherms. J Exp Biol 182:41-56

Hofmann HA, Schildberger K (2001) Assessment of strength and willingness to fight during aggressive encounters in crickets. Anim Behav 62:337-348

Honek A (1993) Intraspecific variation in body size and fecundity in insects: a general relationship. Oikos 66:483-492

Hunt J, Allen GR (2000) Larval density and developmental instability in the acoustically orienting parasitoid Homotrixa alleni. Acta Ethol 2:129-132

Kolluru GR, Zuk M (2001) Parasitism patterns and the sizefecundity relationship in the acoustically orienting dipteran parasitoid Ormia ochracea. Can J Zool 79:973-979

Kolluru GR, Zuk M, Chappell MA (2002) Reduced reproductive effort in male field crickets infested with parasitoid fly larvae. Behav Ecol 13:607-614

Krasnov BR, Burdelov SA, Khokhlova IS, Burdelova NV (2003) Sexual size dimorphism, morphological traits and jump performance in seven species of desert fleas (Siphonaptera). J Zool Lond 261:181-189
Krasnov BR, Khokhlova IS, Burdelov SA, Fielden LJ (2004) Metabolic rate and jump performance in seven species of desert fleas. J Insect Physiol 50:149-156

Lederhouse RC, Morse RA, Ambrose JT, Burgett DM, Conner WE, Edwards L, Fell RD, Rutowski R, Turell M (1976) Crepuscular mating aggregations in certain Ormia and Sitophaga. Ann Entomol Soc Am 69:656-658

Morgan KR (1987) Temperature regulation, energy metabolism and mate-searching in rain beetles (Pleocoma spp.), winter-active, endothermic scarabs (Coleoptera). J Exp Biol 128:107-122

Moulin B, Rybak F, Aubin T, Jallon J-M (2001) Compared ontogenesis of courtship song components of males from the sibling species, D. melanogaster and D. simulans. Behav Genetics 31:299-308

Müller CB, Fritsche F (1993) Unequal costs of parasitism for male and female lygaeid bugs. J Insect Behav 6:265-269

Müller P, Robert D (2001) A shot in the dark: the silent quest of a free-flying phonotactic fly. J Exp Biol 204:1039-1052

Murray A-M, Cade WH (1995) Differences in age structure among field cricket populations (Orthoptera; Gryllidae): possible influence of a sex-biased parasitoid. Can J Zool 73:1207-1213

Odell JP (1998) Energetics of metamorphosis in two holometabolous insect species: Manduca sexta (Lepidoptera: Sphingidae) and Tenebrio molitor (Coleoptera: Tenebrionidae). J Exp Zool 280:344-353

Prestwich KN (1994) The energetics of acoustic signaling in anurans and insects. Am Zool 34:625-643

Reinhold K (1999) Energetically costly behaviour and the evolution of resting metabolic rate in insects. Funct Ecol 13:217-224

Reznick DN, Braun V (1987) Fat cycling in the mosquitofish (Gambusia affinis): fat storage as a reproductive adaptation. Oecologia 73:401-413

Rivers DB, Denlinger DL (1994) Redirection of metabolism in the flesh fly, Sarcophaga bullata, following envenomation by the ectoparasitoid Nasonia vitripennis and correlation of metabolic effects with the diapause status of the host. J Insect Physiol 40:207-215

Roff D (1977) Dispersal in dipterans: its costs and consequences. J Animal Ecol 46:443-456

Rogowitz GL, Chappell MA (2000) Energy metabolism of eucalyptus-boring beetles at rest and during locomotion: gender makes a difference. J Exp Biol 203:1131-1139

SAS Institute (1995) JMP statistics and graphics guide, version 3 SAS Institute, Cary, North Carolina

Sayle MH (1928) The metabolism of insects. Q Rev Biol 3:542-553

Shillington C, Peterson CC (2002) Energy metabolism of male and female tarantulas (Aphonopelma anax) during locomotion. J Exp Biol 205:2909-2914

Simmons LW, Zuk M (1994) Age structure of parasitized and unparasitized populations of the field cricket, Teleogryllus oceanicus. Ethology 98:333-340

Speirs DC, Sherratt TN, Hubbard SF (1991) Parasitoid diets - does superparasitism pay? Trends Ecol Evol 6:22-25

Vinson SB (1990) How parasitoids deal with the immune system of their host: an overview. Arch Insect Biochem Physiol 13:3-27

Zuk M, Simmons LW, Cupp L (1993) Calling characteristics of parasitized and unparasitized populations of the field cricket Teleogryllus oceanicus. Behav Ecol Sociobiol 33:339-343

Zuk M, Simmons LW, Rotenberry JT (1995) Acoustically-orienting parasitoids in calling and silent males of the field cricket Teleogryllus oceanicus. Ecol Entomol 20:380-383

Zuk M, Simmons LW, Rotenberry JT, Stoehr AM (2004) Sex differences in immunity in two species of field crickets. Can J Zool 82:627-634 